\title{
Cardioprotective effect of melatonin and agomelatine on doxorubicin-induced cardiotoxicity in a rat model: an electrocardiographic, scintigraphic and biochemical study
}

\author{
Aygun $\mathrm{H}^{1}$, Gul SS${ }^{2}$ \\ Department of Physiology, Tokat Gaziosmanpasa University, Faculty of Medicine, Tokat, Turkey. \\ hatice_5aygun@hotmail.com
}

\begin{abstract}
AIM: The present study aimed to determine the protective effect of melatonin and agomelatine on DOX-induced cardiotoxicity in rats by electrocardiographic, scintigraphic and biochemical methods.

MATERIALS AND METHODS: Forty-nine male Wistar rats were randomly separated into seven groups; control (CON), doxorubicin (DOX), melatonin (MEL), agomelatine (AGO), melatonin+doxorubicin (MEL+DOX), agomelatine+doxorubicin (AGO+DOX) and melatonin+agomelatine+doxorubicin (MEL+AGO+DOX) groups. Cardiotoxicity was induced by intraperitoneal (i.p.) injection of DOX (18 mg/kg daily for three days). Rats receiving MEL and AGO treatment in the DOX-induced cardiotoxicity group received MEL and AGO (40 mg/kg/day, i.p., for seven days). They were injected with doxorubicin (18 $\mathrm{mg} / \mathrm{kg}$, i.p.) on days 5,6 , and 7 . The rats were given MEL and AGO as substance control (40 mg/kg/day, i.p., for 7 days). On day 8 of the experiment, animals were evaluated by means of electrocardiography (ECG) and ${ }^{99 m}$ technetium pyrophosphate ( ${ }^{99 m} \mathrm{TC}$ PYP) scintigraphy and their biochemical parameters [blood urea nitrogen (BUN), creatine kinase (CK), cardiac troponin T (cTnT)] were examined.

RESULTS: DOX-induced acute cardiotoxicity in rats is characterized by conduction abnormalities in the ECG pattern (including decreased $\mathrm{P}$ wave and QRS complex duration, increased QT and RR intervals, and STsegment elevation), increased serum BUN, CK, and cTnT parameters and increased ${ }^{99 m}$ Tc PYP uptake ( $p<$ 0.001). Pretreatment with MEL, AGO, or MEL+AGO effectively alleviated DOX-induced ECG abnormalities close to normal $(p<0.001)$. Moreover, serum biochemical evidence and ${ }^{99 m} T c$ PYP uptake values demonstrated that pretreatment with MEL, AGO, or MEL+AGO has the same protective effect against the abnormalities produced in the heart by DOX $(p<0.001)$.

CONCLUSIONS: MEL and AGO have a potential protective effect on DOX-induced cardiotoxicity. At the same time, this study suggests that ${ }^{99 m}$ TC PYP is a non-invasive method suitable for early determination of DOX-induced cardiotoxicity (Tab. 3, Fig. 5, Ref. 41). Text in PDF www.elis.sk.

KEY WORDS: melatonin, agomelatine, doxorubicin, cardiotoxicity, rat, ${ }^{99 \mathrm{~m}} \mathrm{Tc}$ pyrophosphate.
\end{abstract}

\section{Introduction}

Doxorubicin (DOX) is an eminently effective chemotherapeutic drug; however, its clinical use is limited because of its severe cardiotoxicity $(1,2)$. DOX-induced cardiac toxicity involves an immense clinical spectrum and is explained by early and late effects. Early effects can be detected by electrocardiogram (ECG) changes, namely ST-segment elevation, arrhythmias and sinus tachycardia. Late indications of DOX administration take form of congestive heart failure and cardiomyopathy $(1,3,4)$. However, the mechanism underlying DOX-induced cardiotoxicity is not clearly

${ }^{1}$ Department of Physiology, Tokat Gaziosmanpasa University, Faculty of Medicine, Tokat, Turkey, and ${ }^{2}$ Department of Nuclear Medicine, Tokat Gaziosmanpasa University, Faculty of Medicine, Tokat, Turkey

Address for correspondence: H. Aygun, Department of Physiology, Faculty of Medicine, Tokat Gaziosmanpasa University, 60030 Tokat, Turkey. Phone: +905399631308 understood. Among various mechanisms, it is generally admitted that DOX induces oxidative stress through enhanced reactive oxygen species (ROS) production and depletion of endogenous antioxidants and this triggers the intrinsic mitochondria-dependent apoptotic pathway in cardiomyocytes $(5,6,7)$.

Many studies have shown that potent antioxidants protect the heart against DOX toxicity by reducing oxidative stress $(8,1)$. Melatonin (MEL) is a secretory product of the pineal gland of all mammals and an important natural antioxidant. It also influences various biological processes such as circadian rhythms, and cardiovascular, neuroendocrine and immune functions. It may also reduce DOX-induced oxidative stress (9). Recent studies provide direct confirmation that melatonin protects against DOX-induced toxicity by inhibiting ROS production. This implies that MEL or MEL receptor agonists might produce protective effects on DOXinduced cardiotoxicity $(1,10,11-12)$.

Agomelatine (AGO) is a melatonergic M1 and M2 receptor agonist and also a serotonergic (5-HT2C) receptor antagonist (13). 
It displays a high affinity for M1 and M2 melatonin receptors and mimics the role of MEL in antioxidant properties (14). Many studies have shown that AGO has a protective effect on brain, kidneys, testes, and ovaries. It protects from ischemia-reperfusion injury by enhancing antioxidant properties. A new study by Jia et al (15), demonstrated that AGO protects the heart against myocardial ischemia-reperfusion injury.

However, the protective effect of AGO on DOX-induced cardiotoxicity has not been examined. Therefore, we have investigated the possible cardioprotective effects of AGO alone and in combination with MEL against DOX-induced cardiotoxicity by electrocardiographic, scintigraphic and biochemical methods.

\section{Materials and methods}

\section{Chemicals}

DOX hydrochloride was purchased from Sandoz Pharmaceutical Industry, Turkey. MEL and AGO were obtained from Sigma-
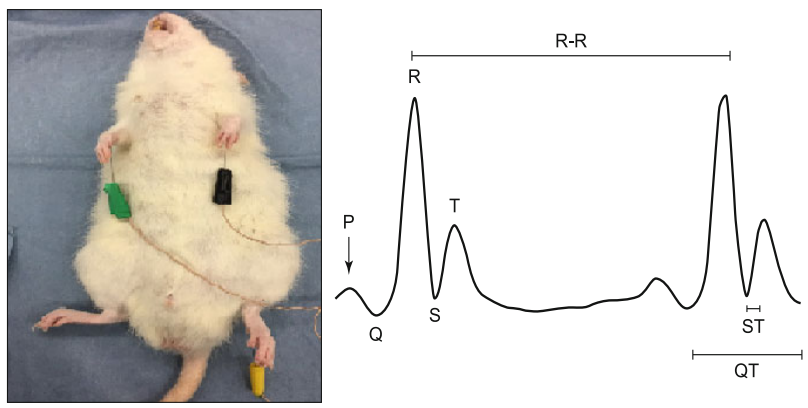

Fig. 1. Limb lead at position II during ECG recording. The negative electrodes are attached to the dermal layers of the front leg paws and the positive electrode is attached to that of the left hind leg paw.

Aldrich Chemicals (St. Louis, MO, USA). MEL and AGO were dissolved in $1 \%$ ethyl alcohol. The required doses were determined in accordance with previous studies and were administered i.p. in a volume of $1 \mathrm{~mL}$ for 7 days $(12,15)$.
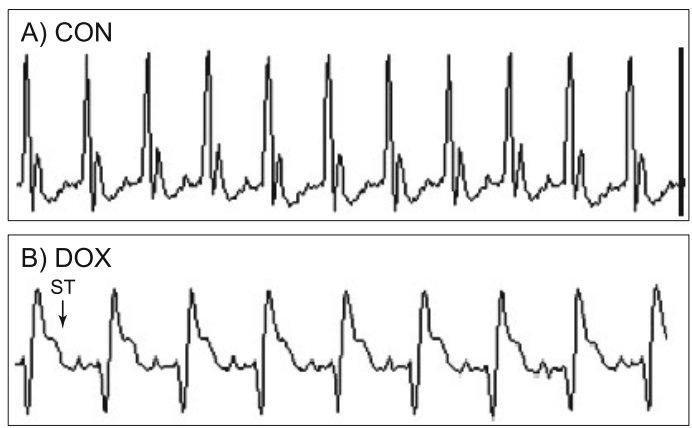

C) MEL + DOX
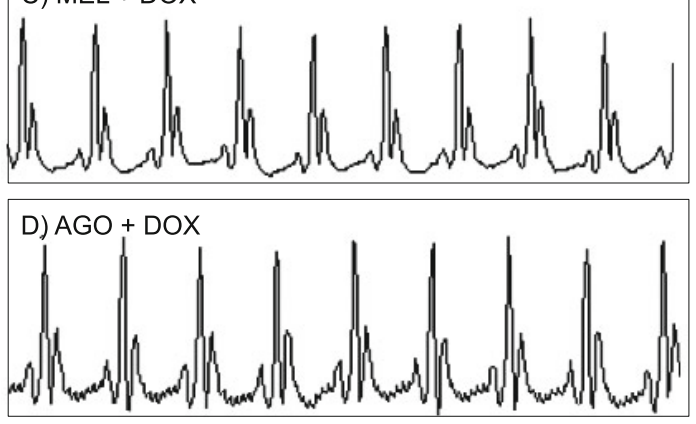

E) $M E L+A G O+D O X$

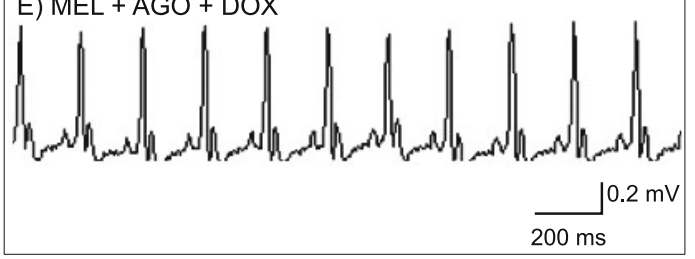

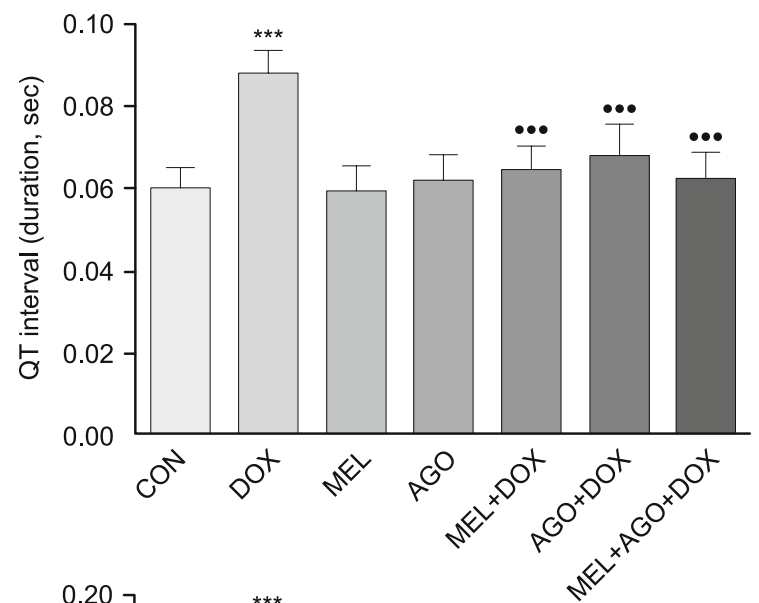

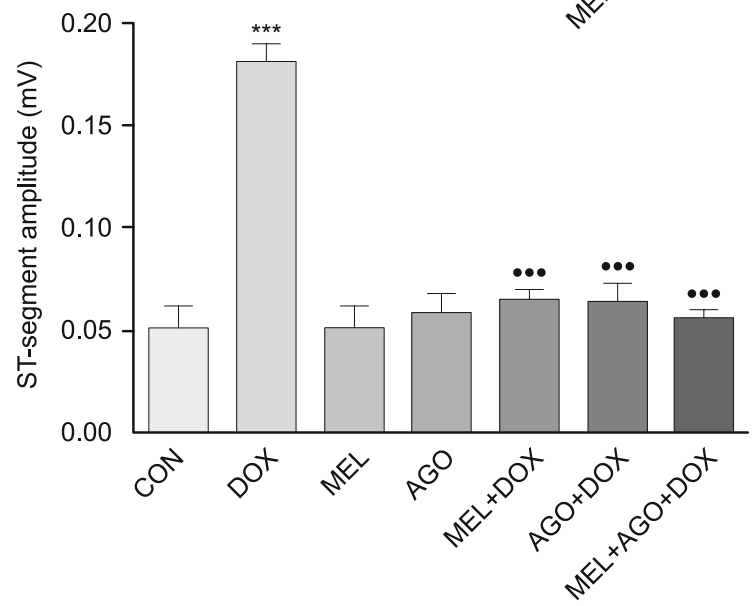

Fig. 2. ECG patterns in the control group (CON, A), doxorubicin administration group (DOX, B), melatonin+doxorubicin group (MEL+DOX, C), agomelatin+doxorubicin group (AGO+DOX, D), and melatonin+agomelatin+doxorubicin group (MEL+AGO+DOX, E). The DOX group had a significant elevation of ST segment amplitude and prolongation of QT interval compared to the control group (***p $<0.001)$ but MEL + DOX, AGO+DOX, and MEL+AGO+DOX did not demonstrate significant changes compared to the control group. Similarly to the CON group, the pre-treatment groups of MEL+DOX, AGO+DOX and MEL+AGO+DOX groups had a significantly decreased ST-segment amplitude and QT interval duration compared to DOX group $(\bullet \bullet \bullet<0.001)$. 
Tab. 1. The statistical analysis of electrocardiographic parameters for all groups.

\begin{tabular}{|c|c|c|c|c|c|}
\hline Groups & $\begin{array}{c}\text { P wave } \\
\text { (duration, sec) }\end{array}$ & $\begin{array}{l}\text { QRS complex } \\
\text { (duration, sec) }\end{array}$ & $\begin{array}{r}\text { QT interval } \\
\text { (duration, sec) }\end{array}$ & $\begin{array}{c}\text { RR interval } \\
\text { (duration, sec) }\end{array}$ & $\begin{array}{l}\text { ST segment amplitude } \\
(\mathrm{mV})\end{array}$ \\
\hline$\overline{\mathrm{CON}}$ & $0.033 \pm 0.01$ & $0.063 \pm 0.02$ & $0.060 \pm 0.01$ & $0.13 \pm 0.01$ & $0.051 \pm 0.03$ \\
\hline DOX & $0.021 \pm 0.08^{\mathrm{c}}$ & $0.033 \pm 0.01^{\mathrm{a}}$ & $0.087 \pm 0.02^{\mathrm{a}}$ & $0.25 \pm 0.00 \mathrm{a}$ & $0.180 \pm 0.03^{\mathrm{a}}$ \\
\hline MEL & $0.034 \pm 0.01$ & $0.061 \pm 0.0$ & $0.059 \pm 0.02$ & $0.13 \pm 0.02$ & $0.051 \pm 0.04$ \\
\hline AGO & $0.035 \pm 0.01$ & $0.058 \pm 0.01$ & $0.061 \pm 0.02$ & $0.14 \pm 0.02$ & $0.058 \pm 0.03$ \\
\hline MEL+DOX & $0.035 \pm 0.01^{\mathrm{b}}$ & $0.059 \pm 0.0^{\mathrm{b}}$ & $0.064 \pm 0.01^{\mathrm{b}}$ & $0.15 \pm 0.01 \mathrm{~b}$ & $0.065 \pm 0.01^{\mathrm{b}}$ \\
\hline $\mathrm{AGO}+\mathrm{DOX}$ & $0.031 \pm 0.02^{\mathrm{b}}$ & $0.062 \pm 0.01^{\mathrm{b}}$ & $0.067 \pm 0.02^{\mathrm{b}}$ & $0.14 \pm 0.01 \mathrm{~b}$ & $0.064 \pm 0.03^{\mathrm{b}}$ \\
\hline
\end{tabular}

$\mathrm{CON}=$ control, $\mathrm{DOX}=$ doxorubicin, $\mathrm{MEL}=$ melatonin, agomelatine $=\mathrm{AGO}$, and MEL $+\mathrm{DOX}=$ melatonin + doxorubicin, $\mathrm{AGO}+\mathrm{DOX}=$ agomelatine + doxorubicin, and $\mathrm{MEL}+\mathrm{AGO}+\mathrm{DOX}=$ melatonin + agomelatine + doxorubicin; ${ }^{\mathrm{a}} \mathrm{p}<0.001$ in all groups as compared to CON groups, ${ }^{\mathrm{b}} \mathrm{p}<0.001 \mathrm{in} \mathrm{MEL}+\mathrm{DOX}, \mathrm{AGO}+\mathrm{DOX}, \mathrm{MEL}+\mathrm{AGO}+\mathrm{DOX}$ groups as compared to DOX groups.

\section{Animals}

Forty-nine adult male Wistar rats, weighing 225-280 g, were used. They were obtained from University of Gaziosmanpaşa Experimental Research Centre. The local ethics committee of Gaziosmanpaşa University (2017/15) approved all experimental procedures. All animals were housed in a temperature-controlled $(23 \pm$ $12^{\circ} \mathrm{C}$ ) environment under a 12 -h light/dark cycle and $50 \%$ humidity, and with free access to tap water and standard laboratory pellet diet.

\section{Experimental design}

The animals were randomly divided into 7 groups of 7 rats per group $(n=7 \times 7)$ :

Group I (Control) served as control group and animals received saline $1 \mathrm{ml} / \mathrm{kg}$ of body weight, i.p, for 7 days.

Group II (DOX) served as DOX group, in which the animals received a total cumulative dose of $18 \mathrm{mg} / \mathrm{kg}$, body weight, i.p. daily at 9:00 a.m. for three days in the study.

Group III (MEL) animals received melatonin treatment (40 $\mathrm{mg} / \mathrm{kg}$ body weight, i.p.) for 7 days.

Group IV (AGO) animals received agomelatine treatment (40 $\mathrm{mg} / \mathrm{kg}$ body weight, i.p.) for 7 days.

Group V (MEL + DOX) animals received melatonin treatment ( $40 \mathrm{mg} / \mathrm{kg}$ body weight, i.p.) for 7 days and were injected with DOX (cumulative dose of $18 \mathrm{mg} / \mathrm{kg}$, i.p.) daily, at 9:00 a.m. on days 5,6 and 7 .

Group VI (AGO + DOX) animals received agomelatine treatment $(40 \mathrm{mg} / \mathrm{kg}$ body weight, i.p.) for 7 days and were injected with DOX (cumulative dose of $18 \mathrm{mg} / \mathrm{kg}$, i.p.) daily, at 9:00 a.m. on days 5,6 and 7 .

Group VII (MEL + AGO + DOX) animals received melatonin and agomelatine treatment $(40 \mathrm{mg} / \mathrm{kg}$ body weight, i.p.) for 7 days and were injected with DOX (cumulative dose of $18 \mathrm{mg} / \mathrm{kg}$, i.p.) daily, at 9:00 a.m. on days 5, 6 and 7.

\section{Electrocardiography}

At the end of the treatment protocol, animals were anesthetized and sedated with a combination of ketamine $(100 \mathrm{mg} / \mathrm{kg}$; i.p. $)+$ xylazine $(10 \mathrm{mg} / \mathrm{kg}$; i.p.). Anesthesia was assessed clinically by pedal reflex. During electrocardiographic (ECG) recordings, rectal temperatures were maintained at $37.5^{\circ} \mathrm{C}$ by a thermostatically controlled heating blanket. In all animals, 10 min after anesthesia, three needle electrodes were inserted under the skin of the animals for the limb lead at position II. As depicted in Figure 1, the lead
II may be achieved in rats by attaching the negative electrodes to the dermal layer of both front leg paws and positive electrode to the left hind leg paw. ECG parameters were recorded for 1 minute by using the MP-150 multi-channel physiological analysis system (MP 150, BIOPAC Systems Inc.; USA). The changes in duration of P wave (sec), QRS complex (sec), QT interval (sec), RR inter$\mathrm{val}(\mathrm{sec})$ and amplitude of ST-segment $(\mathrm{mV})$ were determined.

\section{Scintigraphic images}

$1 \mathrm{mCi}{ }^{99 \mathrm{~m}}$ Technetium pyrophosphate $\left({ }^{99 \mathrm{~m}} \mathrm{Tc}\right.$ PYP) radiopharmaceutical was administered i.v. to anesthetized rats. One hour after ${ }^{99 \mathrm{~m}} \mathrm{Tc}$ PYP radiopharmaceutical administration, static imaging with a dual head gamma camera (E-CAM, Siemens, Germany) was performed in anterior and posterior positions with 2.55 zoom factor. The radionuclide uptake heart area was measured by drawing equal rectangular regions of interest (ROI) and ${ }^{99 \mathrm{~m}} \mathrm{Tc}$ PYP uptake was calculated by the semi-quantitative method to all study groups.

\section{Biochemical assays and measurements of cardiac enzymes}

At the end of the experiment, all rats were deeply anesthetized under light anesthesia and their blood samples were collected into tubes. After $30 \mathrm{~min}$, these tubes were centrifuged at 3,000 rpm for $10 \mathrm{~min}$; blood samples were separated and transferred to Eppendorf tubes for biochemical analysis of creatine kinase (CK), blood urea nitrogen (BUN), and cardiac troponin T (cTnT) levels.

Then the rats were killed under anesthesia.

\section{Statistical analysis}

Statistical analysis was performed using SPSS software (version 17.0; SPSS, USA). The groups of parametric variables were compared by using one-way ANOVA followed by Tukey post hoc test. The groups of nonparametric variables were compared by Kruskal Wallis test. All the values are presented as the mean \pm standard error of the mean (SEM). Graphs were sketched using GraphPad Prism (software, USA) version 7 software. The values of $\mathrm{p}<0.05$ were considered to indicate a statistically significant difference.

\section{Results}

One animal from the DOX-only treated group died prior to the termination of experiment. Pretreatment with $40 \mathrm{mg} / \mathrm{kg}$ i.p. of MEL and AGO totally prevented the mortality. 


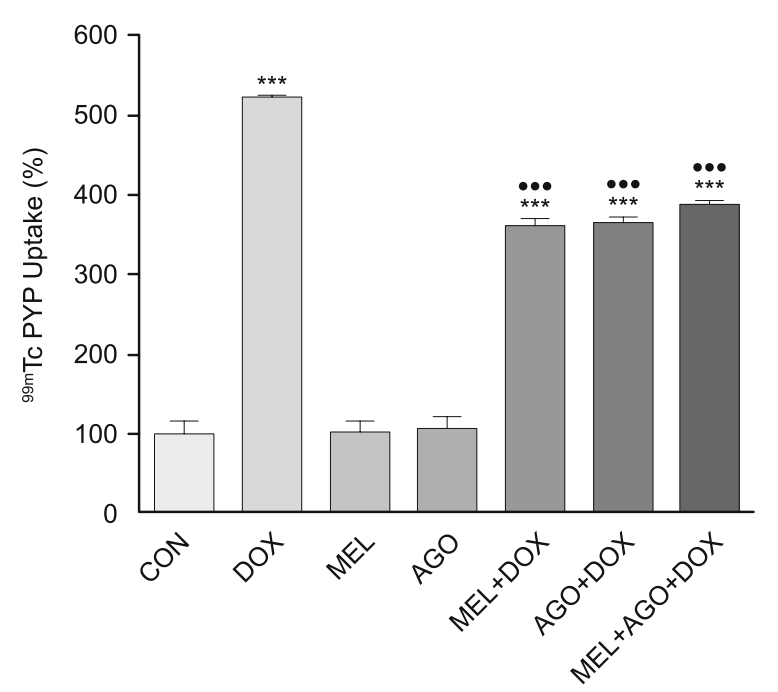

Fig. 4. $\mathrm{CON}=$ control, $\mathrm{DOX}=$ doxorubicin, $\mathrm{MEL}=$ melatonin, agomelatine $=$ AGO, and MEL + DOX $=$ melatonin + doxorubicin, AGO+DOX $=$ agomelatine + doxorubicin, and MEL + AGO+DOX $=$ me latonin+agomelatine+doxorubicin. The MEL+DOX, AGO+DOX, and MEL+AGO+DOX geoups had a significantly decreased ${ }^{99 \mathrm{~m}}$ Tc PYP radiopharmaceutical uptake as compared to the DOX group $(* * * \mathrm{p}<0.001$, $\bullet \bullet p<0.001)$.

\section{Electrocardiography}

Electrocardiographic patterns (P wave duration, QRS complex duration, QT interval, RR interval duration, ST-segment amplitude) of the control and experimental groups are displayed in Figure 2 and Table 1. Rats treated with MEL-only, AGO-only and saline controls group demonstrated a normal pattern on ECG while the DOX-only group, when compared with the control group, displayed a significant decrease in duration of both QRS complex and $P$ wave $(\mathrm{p}<0.001)$, increase in QT interval $(\mathrm{p}<0.001)$, and RR interval $(\mathrm{p}<0.001)$ and elevation in ST-segment amplitude $(\mathrm{p}<0.001)$.

$\mathrm{MEL}+\mathrm{DOX}, \mathrm{AGO}+\mathrm{DOX}$ and MEL+AGO+DOX groups did not demonstrate significant changes in ECG parameters as compared to control group $(\mathrm{p}>0.05)$.

When compared to the DOX group, the MEL+DOX, $\mathrm{AGO}+\mathrm{DOX}$ and $\mathrm{MEL}+\mathrm{AGO}+\mathrm{DOX}$ groups demonstrated a sig-

Tab. 2. The statistical analysis of the scintigraphic data for the all groups.

\begin{tabular}{lc}
\hline Groups & ${ }^{99 m}$ Tc PYP Uptake (cpm) \\
\hline CON & $52,280 \pm 561$ \\
DOX & $273,640 \pm 334^{\mathrm{a}}$ \\
MEL & $52,980 \pm 887$ \\
AGO & $56,280 \pm 621$ \\
MEL+DOX & $188,600 \pm 475^{\mathrm{a}, \mathrm{b}}$ \\
AGO+ DOX & $190,887 \pm 338^{\mathrm{a}, \mathrm{b}}$ \\
MEL+AGO+DOX & $202,960 \pm 292^{\mathrm{a}, \mathrm{b}}$ \\
\hline
\end{tabular}

$\mathrm{CON}=$ control, $\mathrm{DOX}=$ doxorubicin, $\mathrm{MEL}=$ melatonin, agomelatine $=\mathrm{AGO}$, and $\mathrm{MEL}+\mathrm{DOX}=$ melatonin + doxorubicin, $\mathrm{AGO}+\mathrm{DOX}=$ agomelatine + doxorubicin, and $\mathrm{MEL}+\mathrm{AGO}+\mathrm{DOX}=$ melatonin + agomelatine + doxorubicin. Data are as mean \pm SEM. One-way ANOVA and Tukey test. ${ }^{a} \mathrm{p}<0.001$ in all groups as compared to CON groups and ${ }^{b} \mathrm{p}<0.001 \mathrm{MEL}+\mathrm{DOX}, \mathrm{AGO}+\mathrm{DOX}, \mathrm{MEL}+\mathrm{AGO}+\mathrm{DOX}$ groups as compared to DOX groups. nificant increase in duration of both QRS complex and $\mathrm{P}$ wave (Fig. 2 and Tab. 1; $p<0.001$ and $p<0.001$; respectively), and a significant decrease in QT interval, RR interval duration and STsegment-amplitude (Fig. 2 and Tab. 1; $\mathrm{p}<0.001, \mathrm{p}<0.001$, and $\mathrm{p}<0.001$, respectively). As opposed to the DOX group, the pretreatment with MEL and AGO importantly attenuated the DOXinduced abnormalities in ECG parameters.

\section{Scintigraphic images}

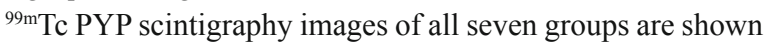
in Figure 3. The DOX-treated group showed an increase in ${ }^{99 \mathrm{~m}} \mathrm{Tc}$ PYP uptake as compared to the CON group. The DOX groups significantly increased the ${ }^{99 \mathrm{~m}} \mathrm{Tc}$ PYP radiopharmaceutical uptake as compared with the CON group (***p $<0.001$, respectively). Pretreatment of DOX-induced toxic rats with MEL, AGO, or combination of MEL and AGO resulted in a significantly decreased ${ }^{99 \mathrm{~m}} \mathrm{Tc}$ PYP radiopharmaceutical uptake, when compared to the DOX group (Fig. 4, Tab. 2; $\mathrm{p}<0.001, \mathrm{p}<0.001$, and $\mathrm{p}<0.001$, respectively).

\section{Biochemical assays}

DOX group demonstrated an increase in BUN $(p<0.001)$, CK $(\mathrm{p}<0.001$, $\mathrm{cTnT}(\mathrm{p}<0.001)$ as opposed to the CON group. The $\mathrm{MEL}+\mathrm{DOX}, \mathrm{AGO}+\mathrm{DOX}$ and $\mathrm{MEL}+\mathrm{AGO}+\mathrm{DOX}$ groups showed a significant decrease in BUN, CK, cTnT (Fig. 5 and Tab. 3; p < $0.001, \mathrm{p}<0.001$, and $\mathrm{p}<0.001$, respectively) when compared to the DOX group.

\section{Discussion}

The electrocardiographic results revealed that DOX-induced cardiotoxicity is associated with ECG abnormalities. In the present study, DOX intoxication significantly decreased the duration of both QRS complex and P wave, increased the RR interval, QT interval and brought about ST segment elevation. In line with the present study, previous studies demonstrated that the duration of P wave (16) and QRS complex $(1,16)$ decreased, while the RR interval duration $(1,17,18,19-20)$ increased. P wave, QRS complex duration, and RR interval are frequently transient and no specificity to DOX-induced toxicity can be assigned to them. Many studies are in agreement with our study by reporting that QT interval prolongation and ST segment elevation brought about by DOX administration have been relatively characteristic ECG findings as a result of DOX-induced cardiotoxicity $(1,16$, $17,18,19,20-21)$. The prolongation of QT interval and ST segment elevation may be linked to the cellular membrane damage owing to oxidative stress $(22,23)$. Treatment with MEL, AGO, and MEL+AGO increased the QRS complex and P wave, and decreased the RR interval, QT interval and ST-segment elevation almost to normal. The ECG changes induced by MEL and AGO might take place owing to their protective effect on myocardium or cell membrane stabilizing action.

Myocardial cells injured by a transient episode of ischemia show an acute marked increase in calcium. Most of this increased calcium is found in mitochondria in form of calcium phosphate 

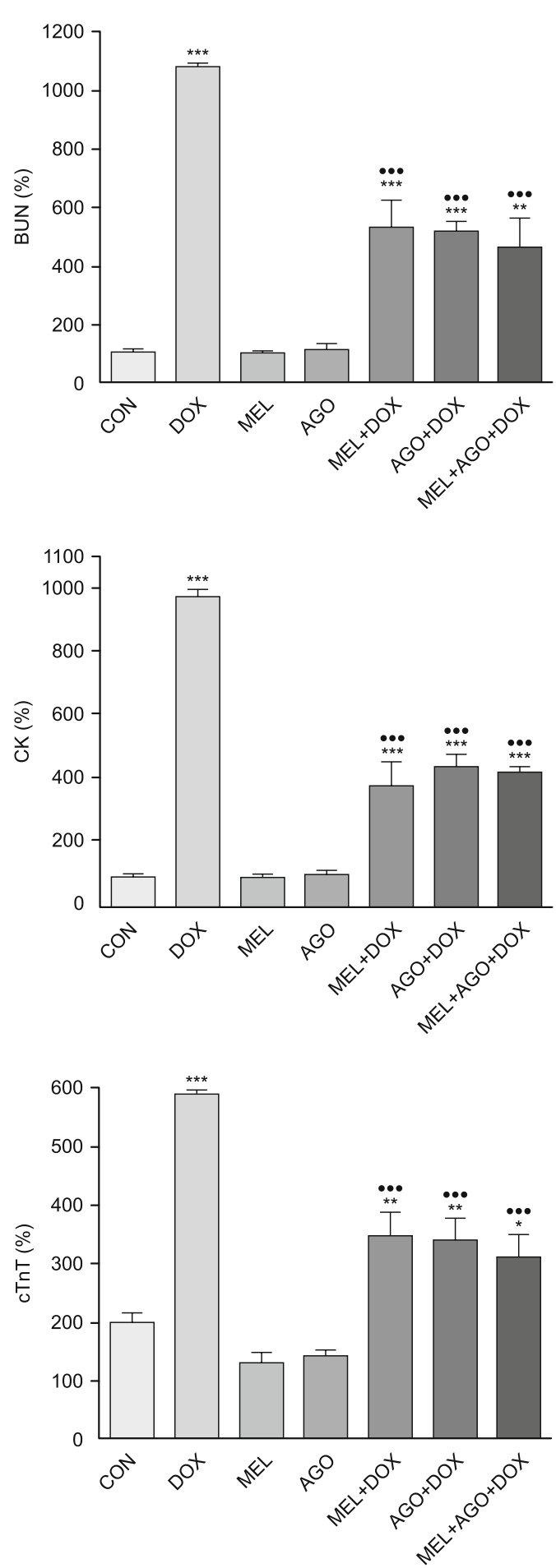

Fig. 5. $\mathrm{CON}=$ control, $\mathrm{DOX}=$ doxorubicin, $\mathrm{MEL}=$ melatonin, $\mathrm{AGO}=$ agomelatine, and MEL+DOX = melatonin+doxorubicin, AGO+DOX $=$ agomelatine + doxorubicin, and $\mathrm{MEL}+\mathrm{AGO}+\mathrm{DOX}=$ melatonin + ago melatine+doxorubicin. The DOX groups had a significant increase in BUN, CK, and cTnT in comparison to the control group $(1076,975$, 294; ***p <.0.001, respectively). Pretreatment of DOX-induced toxic rats with MEL, AGO, or combination of MEL and AGO significantly decreased the levels of BUN, CK and CTnT when compared to the DOX group $(\bullet \bullet \bullet<0.001)$.
Tab. 3. The statistical analysis of the cardiac marker enzyme levels data for the all groups.

\begin{tabular}{lccc}
\hline Groups & BUN & CK & cTnT \\
\hline CON & $16.45 \pm 0.29$ & $0.42 \pm 0.02$ & $3,350 \pm 241$ \\
DOX & $177.1 \pm 2.61^{\mathrm{c}}$ & $4.09 \pm 0.09 \mathrm{c}$ & $9,862 \pm 102^{\mathrm{c}}$ \\
MEL & $15.65 \pm 0.20$ & $0.41 \pm 0.01$ & $2,176 \pm 12,30$ \\
AGO & $18.00 \pm 0.40$ & $0.41 \pm 0.0$ & $2,440 \pm 42,97$ \\
MEL+DOX & $87.25 \pm 15.68^{\mathrm{c}, \mathrm{d}}$ & $1.59 \pm 0.31^{\mathrm{c}, \mathrm{d}}$ & $5,774 \pm 691^{\mathrm{b}, \mathrm{d}}$ \\
AGO+DOX & $85.35 \pm 5.38^{\mathrm{c}, \mathrm{d}}$ & $1.85 \pm 0.17^{\mathrm{c}, \mathrm{d}}$ & $6,106 \pm 660^{\mathrm{b}, \mathrm{d}}$ \\
MEL+AGO+DOX & $75.82 \pm 16.57^{\mathrm{c}, \mathrm{d}}$ & $1.78 \pm 0.05^{\mathrm{c}, \mathrm{d}}$ & $5,201 \pm 622^{\mathrm{a}, \mathrm{d}}$ \\
\hline
\end{tabular}

$\mathrm{CON}=$ control, $\mathrm{DOX}=$ doxorubicin, $\mathrm{MEL}=$ melatonin, agomelatine $=\mathrm{AGO}$, and $\mathrm{MEL}+\mathrm{DOX}=$ melatonin + doxorubicin, $\mathrm{AGO}+\mathrm{DOX}=$ agomelatine + doxorubicin, and $\mathrm{MEL}+\mathrm{AGO}+\mathrm{DOX}=$ melatonin + agomelatine + doxorubicin. Data are as mean $\pm \mathrm{SEM}$. One-way ANOVA and Tukey test. ${ }^{\mathrm{a}} \mathrm{p}<0.05,{ }^{\mathrm{b}} \mathrm{p}<0.01$, and ${ }^{\mathrm{c}} \mathrm{p}<0.001$ in all groups as compared to CON groups; ${ }^{\mathrm{d}} \mathrm{p}<0.001$ in MEL+ DOX, $\mathrm{AGO}+\mathrm{DOX}$, and $\mathrm{MEL}+\mathrm{AGO}+\mathrm{DOX}$ groups as compared to DOX groups.

(24). ${ }^{99 \mathrm{~m}} \mathrm{Tc}$ PYP was originally used in order to image acute myocardial infarcts in both animals and humans. Today, ${ }^{99 \mathrm{~m}} \mathrm{Tc}$ PYP continues to be the most commonly used radiopharmaceutical for myocardial injury because this agent is stable, has good blood clearance properties, and high tagging efficiency, generally gives good bone images and should work equally well $(25,26)$. The uptake of ${ }^{99 \mathrm{~m}} \mathrm{Tc}$ PYP into calcium deposits may have an important role in the concentration of radionuclides in the necrotic myocardium. Myocardial scintigraphy images are generally obtained 60-90 min after injection. Post-injection imaging time needs to be fitted in the "time window" between marked bone uptake and high blood concentration $(27,28)$. Cardiac accumulation patterns of pyrophosphate labeled with ${ }^{99 \mathrm{~m}} \mathrm{Tc}$ in rats one hour to seven days after coronary artery ligation were studied by myocardial scintigraphy, light microscopy, imaging the isolated heart and direct measurement of tissue activity. Results demonstrated that myocardial cells that are taking up ${ }^{99 \mathrm{~m}}$ Tc PYP are irreversibly damaged, and the disappearance of ${ }^{99 m}$ Tc PYP uptake coincides with the removal of necrotic cells by phagocytes (29). In our study, we showed that ${ }^{9 \mathrm{~m}} \mathrm{Tc}$ PYP radiopharmaceuticals uptake was increased in the DOX-induced necrotic myocardium. Treatment with MEL and AGO led to an important decrease in the levels of ${ }^{99 \mathrm{~m}} \mathrm{Tc}$ PYP radiopharmaceutical uptake in the heart area in rats suffering from cardiotoxicity induced with DOX.

Many studies asserted that cardiac lesions induced by DOX could be due to the reactive oxygen species production $(7,30)$, increase in lipid peroxidation and decrease in endogenous antioxidants, thus resulting in increased oxidative stress $(31,32)$. Increased oxidative stress leads to the development of various cellular changes in the myocardium, causing rupture of the cell membrane so that the cellular enzymes leak out (33). Moreover, elevated levels of these biomarker enzymes (BUN, of CK-MB, cTcT ) are an indicator of the severity of DOX-induced myocardial damage and can be estimated in blood samples (34). Studies have demonstrated that DOX-induced cardiotoxicity causes an elevation in levels of these biomarker enzymes (18). The treatment with MEL and AGO caused a significant decrease in the levels of BUN, CK, and cTcT enzymes. It has been stated that MEL displays a capacity for free radical scavenging (35) while this property may disclose a suppression of oxidative stress induced by DOX. On the other hand, 


\section{9-255}

by means of reducing oxidative stress and enhancing antioxidant properties, AGO has been demonstrated to protect brain in cerebral ischemia-reperfusion injury and pentylenetetrazole-induced kindling epilepsy in rats, reduce testicular damage in STZ-induced Type I diabetic rats $(36,37,38-39)$. In our previous study, we showed that Dox-induced anxiety and depression decreased after agomelatine and melatonin treatments (40). AGO is M1, M2 receptors agonist and 5-HT2C receptors antagonist and its affinity for MEL receptors is higher as opposed to the 5-HT2C receptor (14). The repressive effect of AGO on DOX-induced cardiotoxicity was similar to that of MEL.

In this study, electrocardiographic, biochemical and nuclear imaging data indicate that pretreatment with AGO alleviated DOXinduced cardiotoxicity similar to that of MEL. In the early diagnosis of the DOX-induced cardiotoxicity, ECG can be determined as an increase in ST and QT interval and biochemical BUN, CK, and cTcT increase. DOX-induced cardiotoxicity ${ }^{99 \mathrm{~m}} \mathrm{Tc}$ PYP uptake was assessed for this and another study (41). The ${ }^{99 \mathrm{~m}} \mathrm{Tc}$ PYP uptake increase may be helpful in assessing the clinical diagnosis in DOX-induced cardiotoxicity.

\section{Conclusion}

Experimental studies on rats induced with oxidative stress have shown that AGO acts as an antioxidant in the same way as MEL. Therefore, we extensively evaluated the cardioprotective effects of MEL and AGO in DOX-induced cardiotoxicity through various electrophysiologic, scintigraphic, and biochemical parameters. The present study affirmed that a cumulative dose of DOX (18 mg/ $\mathrm{kg} / \mathrm{i} . \mathrm{p}$ ) induces cardiotoxicity in rats as proved by the increase in mortality, ECG changes, increased ${ }^{99 \mathrm{~m}} \mathrm{Tc}$ PYP uptake values and levels of cardiac marker enzymes (BUN, CK, cTcT). Our results demonstrated that MEL and AGO could protect the heart against the undesired effects of DOX.

\section{Limitations}

The fact that the protective effect of melatonin and agomelatine on DOX-induced cardiotoxicity should have been confirmed by histopathological findings is a limitation to our study.

\section{References}

1. Bilginoğlu A, Aydın D, Ozsoy S, Aygün H. Protective effect of melatonin on adriamycin-induced cardiotoxicity in rats. Turk Kardiyol Dern Ars 2014; 42 (3): 265-273.

2. Segredo MP, Salvadori DM, Rocha NS et al. Oxidative stress on cardiotoxicity after treatment with single and multiple doses of doxorubicin. Hum Exp Toxicol 2014; 33: 748-760.

3. Bristow MR, Billingham ME, Mason JW, Daniels JR. Clinical spectrum of anthracycline antibiotic cardiotoxicity. CancerTreat Rep 1978; 62: 873-879.

4. Leonard R, Williams S, Tulpule A et al. Improving the therapeutic index of anthracycline chemotherapy: focus on liposomal doxorubicin (Myocet $^{\mathrm{TM}}$ ). Breast 2009; 18: 218-224.
5. Tokarska-Schlattner M, Zaugg M, Zuppinger C et al. New insights into doxorubicin-induced cardiotoxicity: The critical role of cellular energetics. J Mol Cell Cardiol. 2006; 41 (3): 389-405.

6. Gianni L, Herman EH, Lipshultz SE et al. Anthracycline cardiotoxicity: From bench to bedside. J Clin Oncol. 2008; 26 (22): 3777-3784.

7. Octavia Y, Tocchetti CG, Gabrielson KL et al. Doxorubicin-induced cardiomyopathy: From molecular mechanisms to therapeutic strategies. J Mol Cell Cardiol 2012; 52 (6): 1213-25.

8. Breed JG, Zimmerman AN, Dormans JA, Pinedo HM. Failure of the antioxidant vitamin $\mathrm{E}$ to protect against adriamycin-induced cardiotoxicity in the rabbit. Cancer Res 1980; 40: 2033-2038.

9. Othman AI, El-Missiry MA, Amer MA, Arafa M. Melatonin controls oxidative stress and modulates iron, ferritin, and transferrin levels in adriamycin treated rats. Life Sci 2008; 83 (15-16): 563-568.

10. Liu X, Chen Z, Chua CC et al. Melatonin as an effective protector against doxorubicin-induced cardiotoxicity. Am J Physiol Heart Circ Physiol 2002; 283 (1): 254-263.

11. Kim C, Kim N, Joo H et al. Modulation by melatonin of the cardiotoxic and antitumor activities of adriamycin. J Cardiovasc Pharmacol 2005; 46 (2): 200-210.

12. Zhang Y, Li L, Xiang $\mathbf{C}$ et al. Protective effect of melatonin against Adriamycin-induced cardiotoxicity. Exp Ther Med 2013; 5(5): 1496-1500.

13. Demyttenaere K. Agomelatine: a narrative review. Eur Neuropsychopharmacol 2011; 4: 703-709.

14. Delagrange P, Boutin JA. Therapeutic potential of melatonin ligands. Chronobiol Int 2006; 23: 413-418.

15. Jia $\mathbf{P}, \mathbf{L i u} \mathbf{C}, \mathbf{W u} \mathbf{N}$ et al. Agomelatine protects against myocardial ischemia reperfusion injury by inhibiting mitochondrial permeability transition pore opening. Am J Transl Res 2018; 10 (5): 1310-1323.

16. Koti BC, Nagathan S, Vishwanathswamy A et al. Cardioprotective effect of Vedic Guard against doxorubicin-induced cardiotoxicity in rats: A biochemical, electrocardiographic, and histopathological study. Pharmacogn Mag 2013: 9 (34): 176-181.

17. Tomonari M, To H, Nishida $\mathbf{M}$ et al. Mechanism of the cardioprotective effects of docetaxel pre-administration against adriamycin-induced cardiotoxicity. J Pharmacol Sci 2011; 115 (3): 336-345.

18. Momin FN, Kalai BR, Shikalgar TS, Naikwade NS. Cardioprotective effect of methanolic extract of Ixora coccinea Linn. leaves on doxorubicin-induced cardiac toxicity in rats. Indian J Pharmacol 2012; 44 (2): 178-183.

19. El-Agamy DS, Abo-Haded HM, Elkablawy MA. Cardioprotective effects of sitagliptin against doxorubicin-induced cardiotoxicity in rats. Exp Biol Med 2016; 241 (14): 1577-1587.

20. Bhatt L, Joshi V. Mangifera indica L. leaf extract alleviates doxorubicin induced cardiac stress. J Intercultural Ethnopharmacol 2017; 6 (3): 284.

21. Yarmohmmadi F, Rahimi N, Faghir-Ghanesefat $\mathbf{H}$ et al. Protective effects of agmatine on doxorubicin-induced chronic cardiotoxicity in rat. Eur J Pharmacol 2017; 796: 39-44.

22. Rossi F, Filippelli W, Russo S et al. Cardiotoxicity of doxorubicin: Effects of drugs inhibiting the release of vasoactive substances. Pharmacol Toxicol 1994; 75: 99-107.

23. Holland RP, Brooks H. TQ-ST segment mapping: Critical review and analysis of current concepts. Am J Cardiol 1977; 40: 110-129. 
24. Shen AC, Jennings RB. Kinetics of Calcium Accumulation in Acute Myocardial lschemic Injury. American Journal of Pathology 1972; 62 (3): 441-52.

25. McGregor CG, Hannan J, Smith AF et al. A study of cold cardioplegic myocardial protection in rats: an experimental model using the uptake of technetium $99 \mathrm{~m}$ pyrophosphate and enzyme activity as parameters of injury. Cardiovasc Res 1983; 17 (2): 70-74.

26. Okuda K, Nohara R, Fujita $M$ et al. Improvement of myocardial ischemic dysfunction with dichloroacetic acid: experimental study by repeated ischemia in dogs. J Cardiovasc Pharmacol 1995; 26 (6): 990-999.

27. Parkey RW, Bonte FJ, Buja LM et al. Myocardial infarct imaging with technetium-99m phosphates. Semin Nucl Med 1977; 7 (1): 15-28.

28. Duska F, Hadas L, Volenec K et al. The pathophysiologic basis for the incorporation of $99 \mathrm{mTc}$-pyrophosphate into myocardial infarct. Cesk Radiol 1989; 43 (4): 241-249.

29. Lessem J, Polimeni PI, Page E et al. Accumulation of technetium$99 \mathrm{~m}$ pyrophosphate in experimental infarctions in the rat. Cardiovasc Res 1980; 14 (6): 352-359.

30. Granados-Principal S, El-azem N, Pamplona R et al. Hydroxytyrosol ameliorates oxidative stress and mitochondrial dysfunction in doxorubicin-induced cardiotoxicity in rats with breast cancer. Biochem Pharmacol 2014; 90: 25-33.

31. Fujita T. Formation and removal of reactive oxygen species, lipid peroxides and free radicals, and their biological effects. Yakugaku Zasshi 2002; 122: 203-218.

32. Zhang YW, Shi J, Li YJ, Wei L. Cardiomyocyte death in doxorubicin-induced cardiotoxicity. Arch Immunol Ther Exp (Warsz) 2009; 57 : $435-445$
33. Stark G. Functional consequences of oxidative membrane damage. J Membr Biol 2005; 205: 1-16.

34. Christenson ES, James T, Agrawal V, Park BH. Use of biomarkers for the assessment of chemotherapy-induced cardiac toxicity. Clin Biochem 2015; 48 (4-5): 223-235.

35. Montilla P, Tunez I, Munoz MC et al. Antioxidative effect of melatonin in rat brain oxidative stress induced by adriamycin. Rev Esp Fisiol 1997; 53: 301-305.

36. Yapca OE, Borekci B, Turan MI, Gulapoglu M. The effect of agomelatine on oxidative stress induced with ischemia/reperfusion in rat ovaries. Adv Clin Exp Med 2014; 23: 715-721.

37. Chumboatong W, Thummayot S, Govitrapong P et al. Neuroprotection of agomelatine against cerebral ischemia/reperfusion injury through an antiapoptotic pathway in rat. Neurochem Int 2017; 102: 114-122.

38. Azim MS, Agarwal NB, Vohora D. Effects of agomelatine on pentylenetetrazole-induced kindling, kindling-associated oxidative stress, and behavioral despair in mice and modulation of its actions by luzindole and 1-(m-chlorophenyl) piperazine. Epilepsy \& Behav 2017; 72: 140-144.

39. Yigitturk G, Acara AC, Erbas $O$ et al. The antioxidant role of agomelatine and gallic acid on oxidative stress in STZ induced type I diabetic rat testes. Biomed Pharmacother. 2017; 87: 240-246.

40. Aygun H, Gul SS. Effects of melatonin and agomelatine on doxorubicin induced anxiety and depression-like behaviors in rats. Medical Science and Discovery 2018; 5(7): 253-259.

41. Gul SS, Aygun H. Cardioprotective effect of vitamin D and melatonin on doxorubicin-induced cardiotoxicity in rat model: an electrocardiographic, scintigraphic and biochemical study. The European Res J 2018 Early Online: 1-9. 